\title{
Theorems Relating to Quadratic Forms and their Discriminant Matrices
}

\author{
By S. VAJDA \\ (Received 16th May 1949. Read 4th June 1949.)
}

In a paper read before the Research Branch of the Royal Statistical Society (Ref. 1, p. 150) the following case was considered: Let the expression $\sum_{i=1}^{n}\left(y_{i}-c a_{i}\right)^{2}$ be given; introduce, for $c$, a linear form in $y_{j}$, viz. $c=\sum_{j=1}^{n} b_{j} y_{j}$ and obtain

$$
\sum_{i=1}^{n}\left(y_{i}-a_{i} \sum_{j:=1}^{n} b_{j} y_{j}\right)^{2}
$$

If the $y_{i}$ are sample values from a normal population with unit

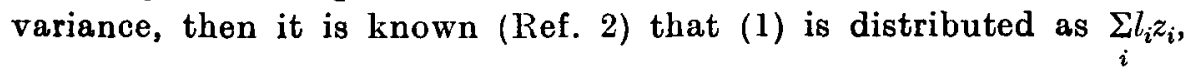
where $z_{i}$ varies as chi-squared with one degree of freedom and the $l_{i}$ are the latent roots of the matrix of the quadratic form. If these latent roots are $f$ times unity and $n-f$ times zero, then this reduces to a chi-squared distribution with $f$ degrees of freedom. It was shown that $\sum_{i} a_{i} b_{i}=1$ was a necessary but not a sufficient condition for such a distribution with $f=n-1$ to arise and it was mentioned that the latter is the case if we take the least-square solution for the $b_{i}$. As will be seen from what follows, the least-square solution is, in fact, the only choice for the $b_{i}$ which leads to a chi-squared distribution with $n-1$ degrees of freedom.

In the following lines we investigate problems related to a more general expression, viz., in matrix notation,

$$
(y-A c)^{\prime}(y-A c)
$$

where $y$ is a column matrix of order $n, c$ a column matrix of order $m$ and $A$ is a matrix of order $n \times m$ and of rank $m$. We introduce $c=B y$, where $B$ is a matrix of order $m \times n$, thus obtaining

$$
(y-A B y)^{\prime}(y-A B y)=y^{\prime} M^{\prime} M y \text {. }
$$

The matrix of the latter quadratic form is seen to be $M^{\prime} M$, where $M$ is defined as $I_{n}-A B$.

The rank of $M$ is the same as that of $M^{\prime} M$, and the first theorem which we shall prove states that

The rank of $M$ (and hence that of $M^{\prime} M$ ) cannot be less than $n-m$. 
We show this by applying a transformation $P^{-1} M P$ with nonsingular $P$, so that the rank of the transformed matrix is the same as that of $M$.

We write $A$ as $\left[\begin{array}{l}A_{1} \\ A_{2}\end{array}\right]$, where $A_{1}$ is a square matrix of order $m \times m$ which may, without loss of generality, be assumed to be non-singular. Similarly, $B=\left[\begin{array}{ll}B_{1} & B_{2}\end{array}\right]$ where $B_{1}$ is square. Let then $P$ be equal to

$$
\left[\begin{array}{ll}
A_{1} & 0 \\
A_{2} & I_{n-m}
\end{array}\right]
$$

and hence $P^{-1}$ equal to

$$
\left[\begin{array}{ll}
A_{1}^{-1} & 0 \\
-A_{2} A_{1}^{-1} & I_{n-m}
\end{array}\right]
$$

We remember that $M=I_{n}-A B=\left[\begin{array}{ll}I_{m}-A_{1} B_{1} & -A_{1} B_{2} \\ -A_{2} B_{1} & I_{n-m}-A_{2} B_{2}\end{array}\right]$

and find

$$
P^{-1} M P=\left[\begin{array}{ll}
I_{m}-B A & -B_{2} \\
0 & I_{n-m}
\end{array}\right] \text {. }
$$

Since the lower right-hand corner is not zero, the rank of this matrix cannot be lower than $n-m$, as was to be proved. It also follows: For $M$ to have precisely rank $n-m$ it is necessary and sufficient that

$$
I_{m}-B A=0 \text {. }
$$

We ask now under what conditions the latent roots of $M^{\prime} M$ are zero ( $m$ times) and unity ( $n-m$ times). Condition (5) is clearly necessary, but not sufficient. A further necessary condition is given by the requirement that the trace (spur) of $M^{\prime} M$ must be $n-m$. More explicitly, we require $\operatorname{tr}\left(I_{n}-A B\right)^{\prime}\left(I_{n}-A B\right)=\operatorname{tr} I_{n}-2 \operatorname{tr} A B+\operatorname{tr}(A B)^{\prime}(A B)=n-m$.

The first term equals $n$ and the second equals $2 m$ in view of (5). Thus (6) reduces to

$$
\operatorname{tr}(A B)^{\prime}(A B)=m .
$$

We can now show that (5) and (7) are sufficient for the discriminant to have as its latent roots $m$ times zero and $n-m$ times unity, by the following argument.

It is known that the least square solution, i.e. that matrix $\hat{B}$ which makes (3) a minimum, has the latent roots just mentioned (Ref. 3). $\hat{B}$ is, in fact, the solution of

$$
A^{\prime} y=A^{\prime} A c=A^{\prime} A \hat{B} y \text {. }
$$


Since this is an identity, we have

$$
\hat{B}=(A \cdot A)^{-1} A^{\prime}
$$

and it will be seen at once that $B^{A}$ satisfies (5) and (7). If we can show that (5) and ( 7 ) admit only one solution, then it must be identical with $\hat{B}$ and hence (5) and (7) are also sufficient to produce the required latent roots. We have therefore to show that

(5) and (7) have only one real solution.

Let one solution be $\hat{B}$ and assume that $\hat{B}+D$ is also a solution. It follows from (5) that

$$
D A=0 .
$$

Furthermore, (7) requires that

$$
\begin{aligned}
\operatorname{tr}[A(\hat{B}+D)]^{\prime}[A(\hat{B}+D)] \\
=\operatorname{tr}(A \hat{B})^{\prime}(A \hat{B})+2 \operatorname{tr}(A D)^{\prime}(A \hat{B})+\operatorname{tr}(A D)^{\prime}(A D)=m .
\end{aligned}
$$

The first term equals $m$ because of (7). The second term is $2 \operatorname{tr} D^{\prime} A^{\prime} A B$ which is equal to $2 \operatorname{tr} D^{\prime} A^{\prime}$ by virtue of (8), and hence zero by virtue of (9). There remains as a condition $\operatorname{tr}(A D)^{\prime}(A D)=0$. It follows that. $A D=0$ and hence $D=0$. This completes the proof.

The theorem just proved can be expressed by saying: there is only one choice of $B$ which leads to a quadratic form having a chisquared distribution with $n-m$ degrees of freedom.

\section{REFERENCES.}

1. S. Vajda, "Statistical investigation of casualties suffered by certain types of vessels," Supp. J. R. S. S., 9 (1947), 141.

2. W. G. Cochran, "The distribution of quadratic forms in a normal system," Proc. Camb. Phil. Soc., 30 (1934), 178.

3. A. C. Aitken, "On least squares and linear combinations of observations," Proc. Roy. Suc. Fdinburgh, 55 (1935), 42.

54 Chapel Way,

EPSOM, SurReY 